\title{
Discrete Morse versus Watershed Decompositions of Tessellated Manifolds
}

\author{
Leila De Floriani, Federico Iuricich, Paola Magillo, and Patricio Simari \\ DIBRIS, University of Genova,Via Dodecaneso n 35, 16146 Genova, Italy \\ Department of Computer Science, University of Maryland, A.V. Williams Bldg, \\ College Park, MD 20740, United States
}

\begin{abstract}
With improvements in sensor technology and simulation methods, datasets are growing in size, calling for the investigation of efficient and scalable tools for their analysis. Topological methods, able to extract essential features from data, are a prime candidate for the development of such tools. Here, we examine an approach based on discrete Morse theory and compare it to the well-known watershed approach as a means of obtaining Morse decompositions of tessellated manifolds endowed with scalar fields, such as triangulated terrains or tetrahedralized volume data. We examine the theoretical aspects as well as present empirical results based on synthetic and real-world data describing terrains and 3D scalar fields. We will show that the approach based on discrete Morse theory generates segmentations comparable to the watershed approach while being theoretically sound, more efficient with regard to time and space complexity, easily parallelizable, and allowing for the computation of all descending and ascending $i$-manifolds and the topological structure of the two Morse complexes.
\end{abstract}

Keywords: Discrete Morse theory, Morse decompositions, watershed, segmentation.

\section{Introduction}

Sensor technology and simulation methods continue to improve, resulting in datasets of growing resolution and richness, and therefore size. This growth in data calls for the investigation of efficient and scalable tools for their analysis. Topological methods, known in spatial data analysis and scientific visualization for their ability to extract essential features from data, are a prime candidate for the development of such tools.

Topological methods are rooted in Morse theory [12, which is the basis for defining decompositions of a manifold endowed with a scalar field into regions of influence of the critical points of the field, called Morse complexes. However, Morse theory applies to smooth functions, while in practical applications we deal with scalar fields that are regularly or irregularly sampled at discrete locations within the domain. Thus, recent research has focused on combinatorial topological methods that are beneficial in the presence of noisy data.

A. Petrosino (Ed.): ICIAP 2013, Part II, LNCS 8157, pp. 339-348, 2013.

(C) Springer-Verlag Berlin Heidelberg 2013 
Forman's discrete Morse theory [5] extends Morse theory to cell complexes. In addition to its theoretical contributions, the discrete formulation has practical applications in avoiding derivative computations, thus permitting the formulation of robust discrete algorithms [7914] that overcome the intrinsic limitations of previous algorithms for approximating Morse complexes. On the other hand, algorithms based on discrete Morse theory have been developed for regular grids [914] or for triangle and tetrahedral meshes of limited size 9]. The motivation is that they require the encoding of all the cells of the mesh as well as of their boundary and co-boundary relations. This will be prohibitive for large simplicial meshes. In our work, we have developed methods for computing discrete Morse complexes on unstructured triangle and tetrahedral meshes, taking the algorithm of Robins et al. 14] (the only algorithm that extracts the minimal number of critical cells in 2D and 3D) and extending it to simplicial meshes with irregular connectivity. In 21, we have developed a compact representation of the Forman gradient field, which we do not report here for brevity.

Here, we compare the validity of the results obtained through the Forman approach with those obtained via watershed, which is one of the best-known algorithms used in image segmentation. Our watershed implementation uses a simulated immersion approach, since our previous investigation on triangulated terrain segmentation based on watershed has shown this is the best alternative 1]. After discussing the differences between these two approaches, we will also examine the two methods empirically, comparing their results on synthetic as well as real-world datasets and measuring the similarity of the results using standard segmentation comparison metrics. We will show that the approach based on discrete Morse theory generates segmentations comparable to the watershed approach while being theoretically sound, more efficient with regard to time and space complexity, easily parallelizable, and allowing for the computation of all descending and ascending $i$-manifolds and the topological structure of the two Morse complexes.

\section{Background}

\subsection{Morse Theory and Morse Complexes}

Morse theory captures the relationship between the topology of a manifold $M$ and the critical points of a real-valued function $f$ defined on $M\left[12\right.$. Let $f$ be a $C^{2}$ real-valued function (scalar field) defined over a manifold $M$. A point $p \in M$ is a critical point of $f$ if and only if the gradient $\nabla f$ vanishes at $p$. Function $f$ is said to be a Morse function if all its critical points are non-degenerate (the Hessian matrix $\operatorname{Hess}_{p} f$ at $p$ is non-singular). The number $i$ of negative eigenvalues of $\mathrm{Hess}_{p} f$ is called the index of critical point $p$. If the index of $p$ is $i, 0 \leq i \leq d, p$ is called an $i$-saddle. A 0 -saddle is a minimum, and a $d$-saddle is a maximum.

An integral line of a function $f$ is a maximal path that is everywhere tangent to the gradient $\nabla f$ of $f$. Each such line follows the direction in which the function has the maximum growth, starting at a critical point of $f$ (its origin) and ending at another critical point (its destination). Integral lines that converge to 


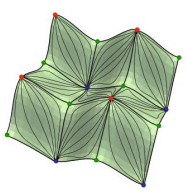

(a)

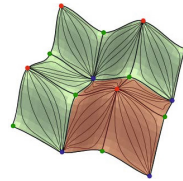

(b)

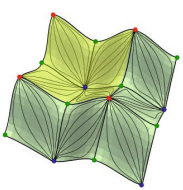

(c)

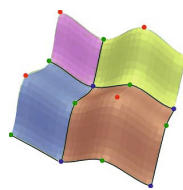

(d)

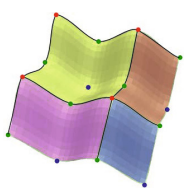

(e)

Fig. 1. Set of the integral lines covering the dataset is shown in (a). In (b) descending 2manifold (red region) formed by the integral lines converging at the maxima (red dot) and dually in (c) ascending 2-manifold formed by integral lines (light green region) originating from the minima (blue dot). The union of all the descending/ascending manifolds forms the descending (d)/ascending (e) Morse complex.

a critical point $p$ of index $i$ cover an $i$-cell called the descending cell of $p$. Dually, integral lines that originate at $p$ cover a $(d-i)$-cell called the ascending cell of $p$. Descending cells decompose $M$ into a cell complex $\Gamma_{d}$, called the descending Morse complex of $f$ on $M$. Dually, the ascending cells form the ascending Morse complex $\Gamma_{a}$ of $f$ on $M$. A maximum corresponds to a $d$-cell in the descending complex $\Gamma_{d}$ and to a 0 -cell in the ascending complex $\Gamma_{a}$. Dually, a minimum corresponds to a 0 -cell in $\Gamma_{d}$ and to an $d$-cell in $\Gamma_{a}$. See Figure 1

\subsection{Discrete Morse Theory}

The main purpose of Discrete Morse Theory (DMT) [5] is to develop a discrete setting in which almost all the main results from smooth Morse theory are valid. This goal is achieved by considering a function $F$ defined on all cells of a cell complex. In the following, we will restrict ourselves to simplicial complexes $\Sigma$, but all definitions and results hold for an arbitrary complex [5].

Function $F$ is a discrete Morse function over $\Sigma$ if for any $i$-simplex $\sigma$ all the $(i-1)$-simplices on the boundary of $\sigma$ have a lower $F$ value than $F(\sigma)$, and all the $(i+1)$-simplices on the co-boundary of $\sigma$ have a higher $F$ value than $F(\sigma)$, with at most one exception. If there is such an exception, it defines a pairing of cells of $\Sigma$ called a discrete (Forman) gradient vector field $V$. Otherwise, $i$-simplex $\sigma$ is a critical simplex of index $i$.

As noted by Forman [5], it is not easy to construct discrete Morse functions; it is simpler to define a discrete gradient vector field. Intuitively, a discrete vector field can be viewed as a collection of arrows, connecting a $p$-simplex of $\Sigma$ to an incident $(p+1)$-simplex of $\Sigma$, such that each simplex is a head or a tail of at most one arrow. A discrete vector field $V$ is a discrete (Forman) gradient vector field if there are no closed $V$-paths in $V$. A $V$-path is a sequence $\sigma_{0}, \tau_{0}, \sigma_{1}, \tau_{1}, \ldots, \sigma_{r+1}$ of $p$-simplices $\sigma_{i}$ and $(p+1)$-simplices $\tau_{j}, i=0, \ldots, r+1, j=0, \ldots, r$, such that $\left(\sigma_{i}, \tau_{i}\right) \in V, \tau_{i}>\sigma_{i+1}$, and $\sigma_{i} \neq \sigma_{i+1}$. Critical simplices for $V$ are those simplices that are neither the head nor the tail of any arrow. Minima are always at vertices while maxima are always at $d$-simplices. In $2 \mathrm{D}$, saddles are on edges, while in $3 \mathrm{D} 1$-saddles occur at edges and 2-saddles are at triangles. 


\section{Related Work}

There are essentially two approaches in the literature to extend the results of Morse theory and represent the Morse complex and the Morse-Smale complex (which is the intersection of the ascending and descending Morse complexes) in the discrete case. The Forman theory approach [5], as described above, considers a discrete Morse function defined on all the simplices that is compatible with the scalar values given at the vertices. The other approach [4] provides an approximation of the Morse-Smale complex by considering a piece-wise linear interpolation of the given scalar field, called a quasi-Morse-Smale complex. The relationship between the piecewise linear and the Forman approaches is discussed in 10, only for the case of 2D scalar fields.

Several algorithms have been developed for computing quasi-Morse complexes in the computational geometry and geographic data processing literature. A complete survey can be found in [1. Algorithms based on discrete Morse theory have been developed for regular grids 7/14] or for triangle and tetrahedral meshes of limited size [9. Parallel algorithms for computing 2D and 3D MorseSmale complexes for large 2D and 3D structured meshes are presented in [16 17. In [6], a memory-efficient implementation of the algorithm in [14 is presented and applied to 3D scalar fields defined over regular grids for efficient persistent homology computation.

An alternative approach to computing ascending and descending Morse complexes is by way of the watershed decomposition. Roerdink et al. present a survey of watershed approaches [15] and classify them into two broad categories: one approach is based on simulated immersion [18]19, and the other uses the idea of topographic distance and basically reduces to computing shortest paths [11. A comparison between watershed approaches and the previously-mentioned piecewise linear approaches can be found in [1].

\section{Computing Morse Complexes}

\subsection{Watershed-Based Approach}

A Morse complex can also be defined using the watershed transform [15, which provides a decomposition of the domain of a smooth function $f$ into regions of influence associated with its critical points. These regions are called catchment basins $(C B)$. The $C B(p)$ of a critical point $p$ is the set of points that are topographically closer to $p$ than to any other critical point of the same type.

The watershed by simulated immersion approach was introduced for segmenting a 2D image into regions of influence of minima 18. The idea is intuitively simple. Let us consider the surface representing the graph of function $f$, drill holes in place of local minima, and insert this surface in a pool of water, building dams to prevent water coming from different minima to merge. The watershed of $f$ is described by these dams, and the CBs of minima are delineated by them. The algorithm has been extended from images to undirected graphs in which 
the field values are associated with nodes. Note that the watershed by simulated immersion algorithm requires sorting the nodes of the graph according to their associated field value, thus making the time complexity of the algorithm $O(n \log n)$, where $n$ is the number of vertices of $\Sigma$. From the point of view of space complexity the algorithm needs an ordered list (or priority queue) with fixed dimension $n$ since all vertices must be enqueued at the start.

We have implemented the watershed by simulated immersion for simplicial meshes of arbitrary dimension with a field value at the vertices. Vertex adjacencies are extracted without the need to explicitly represent the 1-skeleton of the mesh, but rather through a compact representation given by the Indexed data structure with Adjacencies (IA), which encodes only the vertices and the top simplices (triangles in $2 \mathrm{D}$, tetrahedra in $3 \mathrm{D}$ ), and the adjacencies of the $d$-simplices along their common $(d-1)$-simplices (edges in 2D, triangles in $3 \mathrm{D})$.

The watershed algorithm computes the ascending $d$-manifolds, which are the $\mathrm{CBs}$ of the minima. Also, it computes the ascending $d$-manifolds for function $-f$, which are the descending $d$-manifolds of the maxima of function $f$. The latter are collections of vertices. It should be noted that the watershed approach will only compute the $d$-manifolds of the ascending and descending Morse complex as opposed to the $i$-manifolds and their topological relations.

\subsection{An Approach Based on Discrete Morse Theory}

In 14, a dimension-independent algorithm is proposed for constructing a Forman gradient vector field on a cubical complex $K$ with scalar field values given at the vertices, and applications to the $2 \mathrm{D}$ and $3 \mathrm{D}$ images are presented. The algorithm processes the star of each vertex $v$ in $K$ (i.e., the set of cells incident in $v$ ) independently. The optimality results (on the minimal number of critical cells) proven in 14 holds for general cell complexes, and, thus, in particular for simplicial meshes.

Forman Gradient Computation: We have implemented a version of the algorithm for simplicial meshes using the IA data structure described above. The discrete Morse computation algorithm takes as input a simplicial mesh and the field values given at the vertices of the mesh and outputs a list $C$ of the critical simplices as well as the discrete gradient field. This is generated via homotopic expansions of the lower star of each vertex of the input mesh. The lower star of a vertex $v$ consists of the simplices $\sigma$ incident in $v$ such that $\max _{p \in \sigma} f(p)=f(v)$. Since pairing occurs only between simplices in the same lower star, each lower star can be treated independently in time $O(n)$. The resulting discrete vector field is stored by associating a bit vector with each $d$-simplex. In our implementation, this requires 8 or 16 bits per triangle or tetrahedron [21], respectively.

Extracting Morse Complexes: Using the computed Forman gradient, we have developed a collection of algorithms for extracting all ascending and descending $i$-manifolds and for computing all critical cells and the connection between pairs of them given by the critical (separatrix) lines. The collections of all critical cells plus the combinatorial descriptions of all their adjacencies 


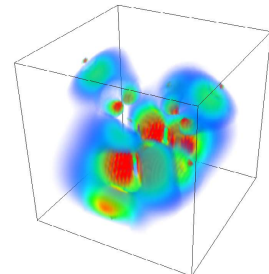

(a)

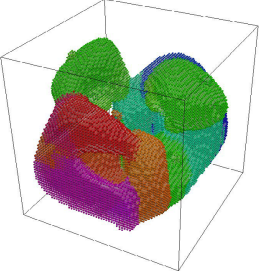

(b)

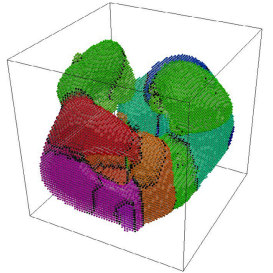

(c)

Fig. 2. Results obtained on the Neghip dataset. A visualization of the field (a), labeling for the vertices obtained computing the ascending 3-manifolds with the Forman approach (b) and with watershed by simulated immersion (c) where black spheres indicate watershed vertices.

completely defines the topology of the two Morse complexes. Generally speaking, a descending or ascending $i$-manifold is extracted starting from the $i$-simplex corresponding to the critical point associated with the $i$-manifold, following the pairings of the gradient field and collecting all the $i$-simplices in the $i$-manifold through a path.

For the sake of comparison with the watershed approach, we focus here on the computation of the ascending and descending $d$-manifolds. We can observe that a descending $d$-manifold corresponds to a maximum and thus to a collection of labeled simplices, while an ascending $d$-manifold corresponds to a minimum and thus to a collection of labeled vertices. We can notice that a maximum of $f$ corresponds exactly to a minimum of $-f$, and thus, that the ascending $d$ manifold extracted on $-f$ corresponds to the descending $d$-manifold on $f$, and is a collection of vertices of the mesh. This allows for a direct comparison with the output of the watershed algorithm, which induces a segmentation over the set of vertices in both the ascending and descending cases.

The ascending $d$-manifold extraction works as follows. For each minimum vertex $p$ the corresponding ascending $d$-manifold is computed by starting from $p$ and initializing a queue $Q$ with it. At a generic step, we extract a vertex $v$ from $Q$ and assign it the label of $p$. Then, for each edge $e$ extracted from the co-boundary of $v$, we check in the gradient if it is paired with a vertex $w$ different from $v$ and, if so, $w$ is enqueued. Since a Forman gradient is always acyclic, the computation terminates and all the vertices are visited exactly once. Figures 2 and 3 present example results.

\section{Discussion and Experimental Results}

The discrete Morse approach pre-computes the gradient field as a preprocessing step, while the watershed approach directly performs the extraction of the $d$ manifolds. On the other hand, with any watershed algorithm we can extract only the ascending and the descending $d$-manifolds as collections of mesh vertices, but not their mutual adjacencies which define the topological structure of the two Morse complexes, or the critical points of the field. The discrete Morse 


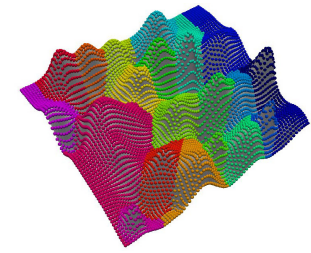

(a)

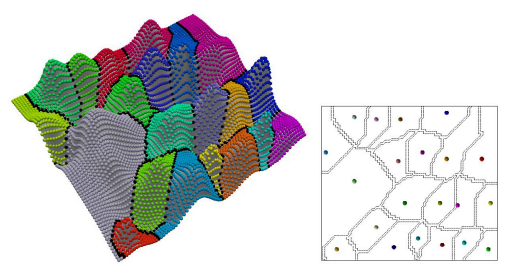

(b)

Fig. 3. Eggs segmentations obtained with the Forman approach (a) and watershed by simulated immersion (b) where black dots correspond to watershed vertices. For each we show the vertex labeling (left) and boundaries and seeds (right). Corridors in the simulated immersion case correspond to unclassified vertices.

approach allows us to compute all descending and ascending $i$-manifolds, and the topological structure of the two Morse complexes. The latter is expressed by the Morse incidence graph [3] in which the nodes are the critical points (simplices in the Forman setting) and the arcs are their adjacencies defined by the discrete counterparts of the separatrix lines. Computing all this information from the output of a watershed algorithm is very inefficient, or, in many cases, incorrect or impossible. There are advantages to the Forman approach. The gradient computation algorithm can be easily parallelized. Further, it is the basis for extracting a complete set of topological features in addition to the Morse incidence graph, which provides a complete description of the topology of the two complexes, and thus it describes the Morse chain complex. This is a suitable basis for computing a homology and persistent homology of 3D and higher dimensional shapes discretized as simplicial or cell complexes.

All datasets used in our experiments satisfy the theoretical conditions that no two adjacent vertices have the same elevation. Therefore, they have no flat regions. For each dataset, we extract the corresponding ascending and descending Morse decomposition using the Forman and watershed approaches and we compare them by using the Rand Index (RI) 13 and the Hamming distance (HD) 8] metrics, two common similarity metrics used to compare image segmentations and adapted in [2] to mesh data. Given two segmentations, the RI measures the probability that a pair of top simplices is either in the same segment in both segmentations, or in different segments in both segmentations. It has the advantage of intuitive appeal but becomes less informative as the number of segments grows, going to 1 in the limit as the second term dominates. The HD first maximizes alignment between two segmentations and then considers the sum cardinality of the symmetric set difference per segment normalized by the total number of top simplices. Here we take the complement to 1 of the metric so that 1 indicates maximum agreement. The metrics have been evaluated considering watershed vertices as neutral, therefore always considering them as labeled in agreement with their counterpart derived by the Forman extraction.

In Table 1 we show results obtained on 15 different datasets. We can notice that the two algorithms compute always the same number of ascending and descending $d$-manifolds. This assures a comparability between the two methods. 


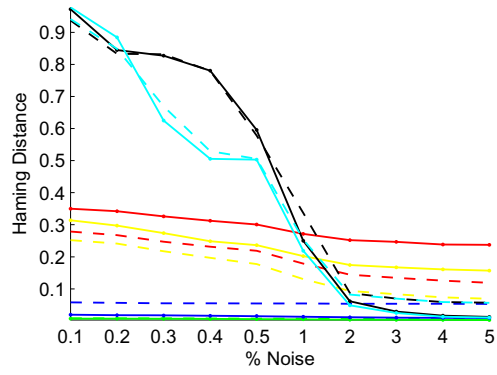

(a)

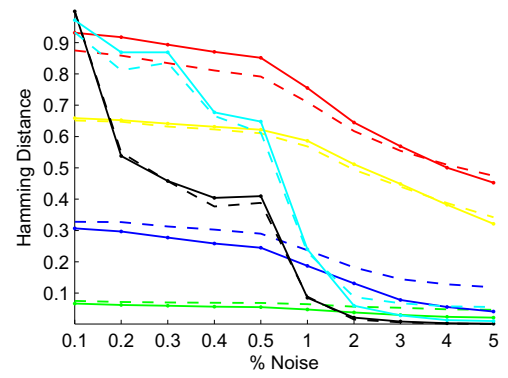

(b)

Fig. 4. HD values for $3 \mathrm{D}$ datasets computed with the Forman (solid) and Watershed (dashed) on the ascending (a) and descending (b) decompositions. Bucky (red), Fuel (green), Neghip (blue), Silicium (yellow), Analytic1 (black), and Analytic2 (cyan).

Table 1. Comparisons between pairs of ascending and descending segmentations found on five terrain, four 3D shapes, and six volume datasets. Columns describe (left to right), dataset name (Name), number of vertices $\left(\left|\Sigma_{0}\right|\right)$, number of triangles/tetrahedra $(T)$, watershed vertices ( $W$. vertices) in the ascending (Asc.) or descending (Desc.) decomposition, number of regions in the watershed $(W$. regions) and in the Forman (F. regions) results, Rand Index value $(R I)$ and Hamming Distance value $(H D)$.

\begin{tabular}{|c|c|c|c|c|c|c|c|c|c|c|c|c|}
\hline \multirow{2}{*}{ Name } & \multirow{2}{*}{$\left|\Sigma_{0}\right|$} & \multirow{2}{*}{$\mathrm{T}$} & \multirow{2}{*}{\multicolumn{2}{|c|}{$\begin{array}{l}\text { W. vertices } \\
\text { Asc. Desc. }\end{array}$}} & \multicolumn{2}{|c|}{ W. regions } & \multicolumn{2}{|c|}{ F. regions } & \multicolumn{2}{|c|}{ RI } & \multicolumn{2}{|c|}{$\mathrm{HD}$} \\
\hline & & & & & Asc. & Desc. & Asc. & Desc. & Asc. & Desc. & Asc. & Des \\
\hline Eggs & 5751 & 11200 & 569 & 567 & 23 & 21 & 23 & 21 & 1.00 & 0.99 & 1.00 & 0.99 \\
\hline St.Helen & 133308 & 265210 & 14433 & 9274 & 572 & 419 & 572 & 419 & 0.99 & 0.97 & 0.82 & 0.94 \\
\hline Monviso & 263169 & 524288 & 30166 & 35349 & 1019 & 1362 & 1019 & 1362 & 0.99 & 0.99 & 0.97 & 0.98 \\
\hline Genova & 433174 & 863059 & 16607 & 13969 & 147375 & 127532 & 147375 & 127532 & 0.99 & 0.99 & 0.93 & 0.97 \\
\hline MajorLake & 810000 & 1616402 & 24494 & 30532 & 257498 & 239292 & 257498 & 239292 & 0.99 & 0.99 & 0.91 & 0.96 \\
\hline Retinal & 3643 & 7282 & 699 & 647 & 44 & 38 & 44 & 38 & 0.99 & 0.99 & 0.99 & 0.99 \\
\hline Camel & 9770 & 19536 & 1712 & 1593 & 93 & 92 & 93 & 92 & 0.99 & 0.99 & 0.98 & 0.99 \\
\hline Bumby Torus & 16815 & 33630 & 1774 & 1593 & 54 & 56 & 54 & 56 & 0.99 & 0.99 & 0.97 & 0.98 \\
\hline Octopus & 16944 & 33872 & 2754 & 3058 & 155 & 186 & 155 & 186 & 0.99 & 0.99 & 0.98 & 0.98 \\
\hline Analytic1 & 68921 & 384000 & 4921 & 0 & 8 & 1 & 8 & 1 & 0.98 & 1.00 & 0.96 & 1.00 \\
\hline Analytic2 & 68921 & 384000 & 4921 & 4921 & 8 & 8 & 8 & 8 & 0.97 & 0.97 & 0.96 & 0.96 \\
\hline Bucky & 32768 & 178746 & 9472 & 14061 & 178 & 223 & 178 & 223 & 0.99 & 0.99 & 0.87 & 0.88 \\
\hline Fuel & 262144 & 1500282 & 1947 & 13075 & 33 & 58 & 33 & 58 & 0.99 & 0.80 & 0.99 & 0.86 \\
\hline Neghip & 262144 & 1500282 & 18423 & 34095 & 88 & 74 & 88 & 74 & 0.79 & 0.95 & 0.80 & 0.77 \\
\hline Silicium & 113288 & 633798 & 19503 & 23862 & 99 & 124 & 99 & 124 & 0.94 & 0.99 & 0.89 & 0.90 \\
\hline
\end{tabular}

Looking at the HD and RI metrics, we found consistently high similarity values, very near to 1, except for the Neghip and Fuel datasets. Such datasets are characterized by a large volume of the scalar field occupied by empty space which can generate small discrepancies between the two methods.

We have also tested the robustness of the two algorithms in 3D, by varying the resolution and adding artificial noise. For each dataset, we extract ten simplifications (decreasing resolution) from a hierarchy of diamond meshes [20], comparing the two methods' output. We observe that the metric values remain similar using the first five/six simplifications and then increase significantly with the final (over-simplified) datasets. The second test was performed independently on both the Forman and watershed methods. For each, we compute the ascending and descending decompositions and then incrementally add Gaussian noise as a percentage of each function's range ( $\max -\min$ value). At each, step we 
evaluate the metrics between the decompositions from the noiseless and noisy datasets. Results obtained on the HD metric are shown in Figure 4 . The RI is more influenced by over-segmentation and became less relevant with noise added. We observe a progressive decay of the metric values different for each dataset. However, values for the Forman method are always equal or better than the simulated immersion values suggesting a better robustness to noise, except for the Fuel and Neghip datasets. Interestingly, as mentioned before, these two datasets are characterized by large volumes of zero values denoting empty space.

\section{Concluding Remarks}

We have compared the new combinatorial approach to segmentation rooted in discrete Morse theory with a common approach based on watershed. We have shown that the discrete Morse theory approach generates segmentations comparable to watershed while being theoretically sound, more efficient in time and space complexity, easily parallelizable, and allowing for the computation of all descending and ascending $i$-manifolds and the topological structure of the two Morse complexes. This last element is the first step for the development of efficient homology and persistent homology algorithms on large-size simplicial meshes. We will tackle this problem in our future work.

We have applied here the two segmentation algorithms to $2 \mathrm{D}$ and $3 \mathrm{D}$ scalar fields defined over a 2D and 3D domain, respectively, and to 3D shapes described by their triangulated boundary with a curvature value associated with their vertices. The same algorithm can be applied with no change to segment $3 \mathrm{D}$ manifolds endowed with a scalar value, like tessellated isosurfaces of time-varying scalar fields by using 3D curvature.

All the algorithms described here as well as our implementations are entirely dimension-independent. The encoding of the discrete gradient field can be generalized to arbitrary dimensions. We will explore this possibility in future work to be able to segment $4 \mathrm{D}$ shapes describing time-varying $3 \mathrm{D}$ scalar fields. Also, since all these algorithms require the field value at each vertex to be different from that of its neighbors, we plan to develop algorithms for the removal of flat areas in the datasets in $2 \mathrm{D}$ and in $3 \mathrm{D}$ and investigate the impact that this would have on the similarity between the extracted decompositions.

Acknowledgements. This work has been partially supported by the National Science Foundation under grant number IIS-1116747, and by the Italian Ministry of Education and Research under the PRIN 2009 program. 2D terrain data is courtesy of the CGIAR consortium for Spatial Information at www.cgiar-csi. org, and the AIM@Shape repository at www.aimatshape.net The 3D volumetric datasets are courtesy of the Volume Library at www9.informatik. uni-erlangen.de/External/vollib. 


\section{References}

1. Biasotti, S., De Floriani, L., Falcidieno, B., Frosini, P., Giorgi, D., Landi, C., Papaleo, L., Spagnuolo, M.: Describing shapes by geometrical-topological properties of real functions. ACM Comput. Surv. 40(4), 12:1-12:87 (2008)

2. Chen, X., Golovinskiy, A., Funkhouser, T.: A benchmark for 3D mesh segmentation. In: Proceedings of ACM SIGGRAPH, p. 73. ACM (2009)

3. Comić, L., De Floriani, L., Iuricich, F.: Dimension-independent multi-resolution morse complexes. Computers and Graphics 36(5), 541-547 (2012)

4. Edelsbrunner, H., Harer, J., Natarajan, V., Pascucci, V.: Morse-Smale Complexes for Piecewise Linear 3-Manifolds. In: Proceedings 19th ACM Symposium on Computational Geometry, pp. 361-370 (2003)

5. Forman, R.: Morse theory for cell complexes. Advances in Mathematics 134, 90-145 (1998)

6. Günther, D., Reininghaus, J., Wagner, H., Hotz, I.: Efficient computation of 3D Morse-Smale complexes and persistent homology using discrete Morse theory. The Visual Computer 28(10), 959-969 (2012)

7. Gyulassy, A., Bremer, P.T., Hamann, B., Pascucci, V.: A practical approach to Morse-Smale complex computation: Scalability and generality. IEEE Transactions on Visualization and Computer Graphics 14(6), 1619-1626 (2008)

8. Huang, Q., Dom, B.: Quantitative methods of evaluating image segmentation. In: Proceedings of the International Conference on Image Processing, vol. 3, pp. 53-56 (1995)

9. King, H.C., Knudson, K., Neza, M.: Generating discrete Morse functions from point data. Experimental Mathematics 14(4), 435-444 (2005)

10. Lewiner, T.: Critical sets in discrete Morse theories: Relating Forman and piecewiselinear approaches. Computer Aided Geometric Design 30(6), 609-621 (2013)

11. Meyer, F.: Topographic distance and watershed lines. Signal Process. 38(1), 113-125 (1994)

12. Milnor, J.: Morse Theory. Princeton University Press, New Jersey (1963)

13. Rand, W.M.: Objective criteria for the evaluation of clustering methods. Journal of the American Statistical Association 66(336), 846-850 (1971)

14. Robins, V., Wood, P., Sheppard, A.: Theory and algorithms for constructing discrete Morse complexes from grayscale digital images. IEEE Transactions on Pattern Analysis and Machine Intelligence 33(8), 1646-1658 (2011)

15. Roerdink, J.B.T.M., Meijster, A.: The watershed transform: definitions, algorithms and parallelization strategies. Fundam. Inf. 41(1-2), 187-228 (2000)

16. Shivashankar, N., Natarajan, V.: Parallel computation of 3D Morse-Smale complexes. Computer Graphics Forum 31(3), 965-974 (2012)

17. Shivashankar, N., Senthilnathan, M., Natarajan, V.: Parallel computation of 2D Morse-Smale complexes. IEEE Transactions on Visualization and Computer Graphics 18(10), 1757-1770 (2012)

18. Soille, P.: Morphological Image Analysis: Principles and Applications, 2nd edn. Springer-Verlag New York, Inc., Secaucus (2003)

19. Vincent, L., Soille, P.: Watershed in digital spaces: An efficient algorithm based on immersion simulation. IEEE Transactions on Pattern Analysis and Machine Intelligence 13(6), 583-598 (1991)

20. Weiss, K., De Floriani, L.: Diamond hierarchies of arbitrary dimension. Computer Graphics Forum (Proceedings SGP 2009) 28(5), 1289-1300 (2009)

21. Weiss, K., Iuricich, F., Fellegara, R., De Floriani, L.: A primal/dual representation for discrete morse complexes on tetrahedral meshes. In: Proceedings Eurovis (2013) 